\title{
Structure of the Set of Bounded Solutions and Existence of Pseudo Almost Periodic Solutions of a Vector Liénard Differential Equation
}

https://doi.org/10.1515/msds-2019-0003

Received September 21, 2018; accepted May 13, 2019

Abstract: We give sufficient conditions ensuring the existence and uniqueness of pseudo almost periodic solution of the vectorial Liénard 's equation.

Keywords: Bounded solutions in the future-almost periodic solutions- pseudo almost periodic solutionsAsymptotically almost periodic function- nonlinear differential equations- Liénard equations- Gradient operator Hessian operator- Strongly monotone opertator- Ergodic function-Symetric matrix - Hessian matrix

\section{Introduction}

In applied sciences, some practical problems concerning mechanics, the engineering techniques fields, economy, control theory, physics, chemistry, biology, medicine, atomic energy, information theory, etc.. are associated with the so called Liénard or modified Liénard's equation. By this time, the qualitative properties of solutions of scalar Liénard or modified Liénard's equation have been intensively discussed, see the references therein.

Liénard equation has been investigated by many authors from various points of view. One of the reason why many mathematicians have been studied this kind of equations is that a broad class of phenomena in sciences and engineering is presented by Liénard's equation.

However, to the best of our knowledge from the literature, the pseudo almost periodicity of solutions for vector Liénard's equation has not been discussed in the literature up to now.

In this work, we study some properties of bounded and pseudo almost periodic solutions of the vector Liénard's equation. More exactly, we study some of the properties of bounded, asymptotically almost periodic or pseudo almost periodic solutions of the following Liénard system:

$$
x^{\prime \prime}+\frac{d}{d t}[\nabla F(x)]+G(x)=p(t) .
$$

where $p: \mathbb{R} \longrightarrow \mathbb{R}^{N}$ is continuous, bounded, pseudo almost periodic function.

$F: \mathbb{R}^{N} \rightarrow \mathbb{R}$, and $G: \mathbb{R}^{N} \rightarrow \mathbb{R}^{N}$.

\footnotetext{
^Corresponding Author: E. Ait Dads: Université Cadi Ayyad, Département de Mathématiques, Faculté des Sciences, B.P. 2390 Marrakech, Morocco, E-mail: aitdads@uca.ma

Unité associée au CNRST ( Morocco) URAC 02.

UMI- UMMISCO 209 ( IRD- UPMC), France

P. Cieutat: Laboratoire de Mathématiques de Versailles, Université de Versailles Saint-Quentin en Yvelines, 45 Avenue des Etats-Unis, 78035 Versailles Cedex, France, E-mail: Philippe.Cieutat@math.uvsq.fr

L. Lhachimi: Université Cadi Ayyad, Département de Mathématiques, Faculté des Sciences, B.P. 2390 Marrakech, Morocco,

E-mail: 1llahcen@gmail.com
} 
On the other hand, the Liénard's equation with a periodic forcing term $p$ has also been studied by many authors. It is known that under some assumptions, the Liénard's problem has multiple periodic solutions and sometimes the dynamics of the solutions are chaotic. We will see that under suitable conditions, $n-$ dimensional Liénard's equation has pseudo almost periodic solutions, in the case where $p(t)$ is so.

The space $\mathbb{R}^{N}$ is endowed with its usual inner product

$\langle x \mid y\rangle:=\sum_{i=1}^{N} x_{i} y_{i}$ and $\|$.$\| denotes the associated Euclidean norm. Denote by \nabla F(x)\left(\right.$ resp. $\left.\nabla^{2} F(x)\right)$ the gradient (resp. the Hessian) of $F$ at a point $x$.

The model of equation. (1) is

$$
x^{\prime \prime}+c x^{\prime}+\frac{1}{x^{\alpha}}=p(t)
$$

where $c \geq 0, \alpha>0$ and $p: \mathbb{R} \longrightarrow \mathbb{R}$ is an almost periodic function, that appears when the restoring force is a singular nonlinearity which becomes infinite in zero. Martínez-Amores and Torres in [18], then Campos and Torres in [7] described the dynamics of equation. (1) in the periodic case, namely the forcing term $p$ is periodic. Recall that the existence of periodic solutions of equation. (1) without friction term $(F=0)$ is proved by Lazer and Solimini in [17] and by Habets and Sanchez in [15] for some Liénard's equations with singularity, more general than equation. (1). In [7], Campos and Torres proved that the existence of a bounded solution on $(0,+\infty)$ implies the existence of a unique periodic solution that attracts all bounded solutions on $(0,+\infty)$. Besides they state that the set of initial conditions of bounded solutions on $(0,+\infty)$ is the graph of a continuous nondecreasing function. Then Cieutat [9] extended results of the paper [7] to the almost periodic case. In [7], the authors used topological tools, such as free homeomorphisms (c.f. [6]), together with truncation arguments. The homeomorphisms used in [7], are the Poincare operators of equation (1), therefore these topological tools are not adapted to the almost periodic case. In [9], the methods used are essentially the recurrence property of the almost periodic functions.

A well known extension of almost periodicity is the notion of asymptotically almost periodicity historically due to Fréchet (c.f. [14] for details). In [20], Zhang introduced an other extension of the almost periodic functions, the so-called pseudo almost periodic functions. In [21], Zhang investigated the existence of pseudo almost periodic solution for a pseudo almost periodic nonlinear perturbation of a linear autonomous ordinary differential equations. Ait Dads and Arino [4] introduced the generalized pseudo almost periodic functions and extended Zhang's results. More details on the concept of pseudo almost periodicity can be found in [2, 4, 21 ]. The question of pseudo almost periodic solutions to some differential equations has been studied by various authors $[3,5,12,16,19,22]$ and references therein.

Our aim is to extend the main results of the paper [1] to the bounded or pseudo almost periodic solutions in vector case. Notably, we describe the set of initial conditions of the bounded solutions on $(0,+\infty)$ and we state some results of the existence of pseudo almost periodic solutions.

The paper is organized as follows: in section 2, we give some preliminary results with the problem in general case. In section 3, we study the structure of solutions that are bounded in the future, when the second member $p$ is bounded. The main result of this section (Theorem 16) extends a result in the almost periodic case [Theorem 2.2, [9]] to the bounded case. To establish this last result, we state some results of comparison of bounded solutions. In section 4, we consider some particular cases, the first one is when $F$ is quadratic and in the second subsection, we are concerned with the case that $G$ is a linear function. We establish that all solutions that are bounded in the future, are asymptotically almost periodic, when the second member $p$ is asymptotically almost periodic. In fact, we state this result for a larger class of second member $p$. Then in the section 5 , we study the existence of pseudo almost periodic solutions. 


\section{General case}

From a convex open subset $\Omega$ of $\mathbb{R}^{N}$, a $C^{2}$ function $F: \Omega \longrightarrow \mathbb{R}$ and two continuous functions: $G: \Omega \longrightarrow \mathbb{R}^{N}$ and $p: \mathbb{R} \longrightarrow \mathbb{R}^{N}$, we consider the following forced vector Liénard's equation :

$$
x^{\prime \prime}+\frac{d}{d t}[\nabla F(x)]+G(x)=p(t) .
$$

We assume that $\nabla^{2} F$ and $G$ are locally lipschitzian and that the following hypotheses hold:

(H1) $-G$ is strictly monotone on $\Omega$, namely : $\left\langle G\left(x_{2}\right)-G\left(x_{1}\right)\right| x_{1}-x_{2}>>0$ for all $x_{1}$ and $x_{2} \in \Omega$ such that $x_{1} \neq x_{2}$.

(H2) $F$ is convex on $\Omega$.

(H3) $\sup _{t \in \mathbb{R}}\|p(t)\|<+\infty$.

Definition 1. A solution $x:(c,+\infty) \longrightarrow \mathbb{R}^{N}$ (with $-\infty \leq c<+\infty$ ) of equation (3) is said to be a solution which is bounded in the future, if there exist a compact $K \subset \Omega$ and $t_{0}>c$ such that $x(t) \in K$, for all $t \geq t_{0}$.

Definition 2. A solution $x: \mathbb{R} \longrightarrow \mathbb{R}^{N}$ of equation. (3) is said to be a bounded solution over $\mathbb{R}$, if there exists a compact $K \subset \Omega$ such that $x(t) \in K$, for all $t \in \mathbb{R}$.

Lemma 3. (Lemma 3.2, [[10]]). Under the hypothesis (H2); If there exist $x$ and $y \in \mathbb{R}^{N}$ such that

$$
\langle\nabla F(x)-\nabla F(y) \mid x-y\rangle=0,
$$

then

$$
\nabla F(x)=\nabla F(y)
$$

Lemma 4. Under the Hypothesis (H2), let $x_{1}$ and $x_{2}$, be two bounded in the future and continuous functions. If

$$
\lim _{t \rightarrow+\infty}<\nabla F\left(x_{1}(t)\right)-\nabla F\left(x_{2}(t)\right) \mid x_{1}(t)-x_{2}(t)>=0,
$$

then

$$
\lim _{t \rightarrow+\infty}\left\|\nabla F\left(x_{1}(t)\right)-\nabla F\left(x_{2}(t)\right)\right\|=0 .
$$

Proof. Suppose the contrary. In this case, there exist a sequence $\left(t_{n}\right)_{n}$ and $\varepsilon>0$ such that $\lim _{n \rightarrow+\infty} t_{n}=+\infty$, and

$$
\text { for all } n \in \mathbb{N},\left\|\nabla F\left(x_{1}\left(t_{n}\right)\right)-\nabla F\left(x_{2}\left(t_{n}\right)\right)\right\| \geq \varepsilon .
$$

Since the two sequences $\left(x_{1}\left(t_{n}\right)\right)$ and $\left(x_{2}\left(t_{n}\right)\right)$ are with values in a compact $K$, they have respectively an adhesion value $x_{1}^{\star}$ and $x_{2}^{\star}$. From (4) and (6), we deduce that

$$
\left\langle\nabla F\left(x_{1}^{\star}\right)-\nabla F\left(x_{2}^{\star}\right) \mid x_{1}^{\star}-x_{2}^{\star}\right\rangle=0 \text { and }\left\|\nabla F\left(x_{1}^{\star}\right)-\nabla F\left(x_{2}^{*}\right)\right\| \geq \varepsilon .
$$

By using Lemma 3, we obtain a contradiction: $\nabla F\left(x_{1}^{\star}\right)=\nabla F\left(x_{2}^{\star}\right)$; then the relation (5) is established.

Definition 5. [22] A function $f: \mathbb{R} \rightarrow \mathbb{E}$ is called ergodic if $f \in \mathcal{C}_{b}(\mathbb{R}, \mathbb{E})$, and $\lim _{r \rightarrow \infty} \frac{1}{2 r} \int_{-r}^{r}\|f(s)\| d s=0$, or equivalently:

$$
\text { for all } \varepsilon>0, \lim _{r \rightarrow+\infty} \frac{1}{2 r} \operatorname{meas}\{t \in[-r, r] ;|f(t)| \geq \varepsilon\}=0 .
$$

Lemma 6. Under the hypothesis (H2), if $x_{1}$ and $x_{2}$, are two bounded and continuous functions. If

$$
t \longrightarrow<\nabla F\left(x_{1}(t)\right)-\nabla F\left(x_{2}(t)\right) \mid x_{1}(t)-x_{2}(t)>
$$

is ergodic, then $t \rightarrow\left\|\nabla F\left(x_{1}(t)\right)-\nabla F\left(x_{2}(t)\right)\right\|$ is also ergodic. 
Proof. There exists a compact $K$ of $\mathbb{R}^{N}$ which contains the ranges of $x_{1}$ and $x_{2}$. Let $\varepsilon>0$. Denoting

$$
K_{\varepsilon}:=\{(u, v) \in K ;\|\nabla F(u)-\nabla F(v)\| \geq \varepsilon\} .
$$

The function $(u, v) \longrightarrow<\nabla F(u)-\nabla F(v) \mid u-v>$ is continuous and nonnegative on the compact $K_{\varepsilon}$ (c.f. Lemma 3), so there exists $\delta>0$ such that

$$
\langle\nabla F(u)-\nabla F(v)| u-v>\geq \delta \text { for all }(u, v) \in K_{\varepsilon} .
$$

We deduce the inclusion of the following ergodic sets :

$$
\begin{aligned}
& \left\{t \in[-T, T], \quad\left\|\nabla F\left(x_{1}(t)\right)-\nabla F\left(x_{2}(t)\right)\right\| \geq \varepsilon\right\} \\
& \subset\left\{t \in[-T, T], \quad\left\langle\nabla F\left(x_{1}(t)\right)-\nabla F\left(x_{2}(t)\right)\right| x_{1}(t)-x_{2}(t)>\geq \delta\right\},
\end{aligned}
$$

and consequently the ergodicity of

$$
t \longrightarrow<\nabla F\left(x_{1}(t)\right)-\nabla F\left(x_{2}(t)\right) \mid x_{1}(t)-x_{2}(t)>,
$$

implies the ergodicity of $t \longrightarrow\left\|\nabla F\left(x_{1}(t)\right)-\nabla F\left(x_{2}(t)\right)\right\|$.

Lemma 7. Under the hypothesis (H1), if $x_{1}$ and $x_{2}$, are two continuous and bounded functions in the future such that

$$
\lim _{t \rightarrow+\infty}<G\left(x_{1}(t)\right)-G\left(x_{2}(t)\right) \mid x_{1}(t)-x_{2}(t)>=0,
$$

then

$$
\lim _{t \rightarrow+\infty}\left\|x_{1}(t)-x_{2}(t)\right\|=0
$$

Proof. It suffices to apply Lemma 4 and Lemma 6 to $S(x)=\nabla F(x)$ and $S(x)=G(x)$.

Lemma 8. Under the hypothesis (H1), if $x_{1}$ and $x_{2}$, are two continuous and bounded functions over $\mathbb{R}$ such that $t \longrightarrow<G\left(x_{1}(t)\right)-G\left(x_{2}(t)\right) \mid x_{1}(t)-x_{2}(t)>$ is ergodic, then $x_{1}-x_{2}$ is ergodic too.

Proof. It suffices to apply Lemma 4 and Lemma 6 to $S(x)=\nabla F(x)$ and $S(x)=G(x)$.

Lemma 9. Under the hypothesis (H3), let $\mathbf{I}=\left(t_{0},+\infty\right)$ with $t_{0}=-\infty$ or $t_{0} \in \mathbb{R}$. If $x$ is a solution of equation (3) which is bounded in the future (resp. bounded on $\mathbb{R}$ ), i.e. $x(t) \in K$ for all $t>t_{0}>-\infty$ (resp. $\left.t \in \mathbb{R}\right)$, then $x^{\prime}$ and $x^{\prime \prime}$ satisfy $\sup _{t \in I}\left\|x^{\prime}(t)\right\| \leq c_{1}<+\infty$ and $\sup _{t \in I}\left\|x^{\prime \prime}(t)\right\| \leq c_{2}<+\infty$, where

$$
c_{1}:=\frac{1}{2} \sup _{t \in \mathbb{R}}\|p(t)\|+\frac{1}{2} \sup _{z \in K}\|G(z)\|+2 \sup _{z \in K}\|\nabla F(z)\|+2 \sup _{t \in I}\|x(t)\|<+\infty,
$$

and

$$
c_{2}:=\sup _{t \in I}\|p(t)\|+\sup _{z \in K}\|G(z)\|+c_{1} \sup _{z \in K}\left\|\nabla^{2} F(z)\right\|<+\infty .
$$

Proof. Let $t \in \mathbf{I}$. By Taylor's formula, we obtain that

$$
x(t+1)=x(t)+x^{\prime}(t)+\int_{0}^{1}(1-\theta) x^{\prime \prime}(t+\theta) d \theta,
$$

thus

$$
\left\|x^{\prime}(t)\right\| \leq 2 \sup _{t \in I}\|x(t)\|+\left\|\int_{0}^{1}(1-\theta) x^{\prime \prime}(t+\theta) d \theta\right\| .
$$


From equation (3), we deduce that

$$
\begin{aligned}
& \left\|\int_{0}^{1}(1-\theta) x^{\prime \prime}(t+\theta) d \theta\right\| \leq \frac{1}{2}\left(\sup _{t \in \mathbb{R}}\|p(t)\|+\sup _{z \in \mathbb{R}}\|G(z)\|\right) \\
& +\left\|\int_{0}^{1}(1-\theta) \frac{d}{d \theta}[\nabla F(x(t+\theta))] d \theta\right\|,
\end{aligned}
$$

by integrating by parts, we obtain

$$
\int_{0}^{1}(1-\theta) \frac{d}{d t}[\nabla F(x(t+\theta))] d \theta=-\nabla F(x(t))+\int_{0}^{1} \nabla F(x(t+\theta)) d \theta,
$$

so

$$
\left\|\int_{0}^{1}(1-\theta) \frac{d}{d t}[\nabla F(x(t+\theta))] d \theta\right\| \leq 2 \sup _{z \in K}\|\nabla F(z)\| .
$$

By (9)-(11), one has

$$
\left\|x^{\prime}(t)\right\| \leq c_{1}, \quad \text { for all } t \in \mathbf{I},
$$

where $c_{1}$ is defined by (7). By equation (3), we deduce

$$
\left\|x^{\prime \prime}(t)\right\| \leq c_{2}, \quad \text { for all } t \in \mathbf{I},
$$

where $c_{2}$ is the constant defined by (8).

Let $c \in \mathbb{R}$. Denoting $\mathcal{E}^{+}\left((c,+\infty), \mathbb{R}^{N}\right)$ the set defined by $p \in \mathcal{E}^{+}\left((c,+\infty), \mathbb{R}^{N}\right)$ if and only if $p \in C_{b}\left((c,+\infty), \mathbb{R}^{N}\right)$ and $\lim _{n \rightarrow+\infty} \int_{n}^{n+1}\|p(t)\| d t=0$.

Lemma 10. Under the hypothesis (H3), assume that the second member $p$ of equation (3) is the sum of $p=$ $p^{a p}+p^{e}$ with $p^{a p} \in A P\left(\mathbb{R}, \mathbb{R}^{N}\right)$ and $p^{e} \in \mathcal{E}^{+}\left((c,+\infty), \mathbb{R}^{N}\right)$. Let $x$ be a solution which is bounded in the future of equation (3). If there exist a real sequence $\left(t_{n}\right)_{n}$ and $p_{\star}^{a p} \in A P\left(\mathbb{R}, \mathbb{R}^{N}\right)$ such that

$$
\lim _{n \rightarrow+\infty} t_{n}=+\infty
$$

and

$$
\lim _{n \rightarrow+\infty} \sup _{t \in \mathbb{R}}\left\|p^{a p}\left(t+t_{n}\right)-p_{\star}^{a p}(t)\right\|=0 .
$$

Then there exists a subsequence of $\left(t_{n}\right)_{n}$ such that

$$
\text { for all } t \in \mathbb{R}, \quad \lim _{n \rightarrow+\infty} x\left(t+t_{n}\right)=x_{\star}(t),
$$

where $x_{\star}$ is a bounded solution over $\mathbb{R}$ of the following equation

$$
\chi_{\star}^{\prime \prime}+\frac{d}{d t}\left[\nabla F\left(\chi_{\star}\right)\right]+G\left(x_{\star}\right)=p_{\star}^{a p}(t)
$$

Proof. Since $x$ is a bounded solution in the future, then there exists $t_{0} \in \mathbb{R}$ such that

$$
x(t) \in K \quad \text { for all } t \geq t_{0} .
$$

From Lemma (9), we obtain

$$
\sup _{t>t_{0}}\left\|x^{\prime}(t)\right\| \leq c_{1}<+\infty
$$


and

$$
\sup _{t>t_{0}}\left\|x^{\prime \prime}(t)\right\| \leq c_{2}<+\infty .
$$

Let $(\tau,+\infty)$, for $n \in \mathbb{N}$ be large enough $\left(\tau+t_{n} \geq t_{0}\right)$. Then the function $t \longrightarrow x\left(.+t_{n}\right)$ is defined on $(\tau,+\infty)$ and from (17), we have

$$
x\left(t+t_{n}\right) \in K \quad \text { for all } t \in(\tau,+\infty) .
$$

From (18) and (19), we deduce

$$
\begin{array}{ll}
\left\|x^{\prime}\left(t+t_{n}\right)\right\| \leq c_{1} & \text { for all } t \in(\tau,+\infty), \\
\left\|x^{\prime \prime}\left(t+t_{n}\right)\right\| \leq c_{2} & \text { for all } t \in(\tau,+\infty) .
\end{array}
$$

By considering $\left(t_{n}\right)_{n}$ as a sequence which goes to $-\infty$. From Ascoli's Theorem and diagonalization principle, we can assert the existence of $x_{\star} \in C^{1}\left(\mathbb{R}, \mathbb{R}^{N}\right)$ and a subsequence of $\left(t_{n}\right)_{n}$ such that

$$
\begin{aligned}
& x\left(t+t_{n}\right) \rightarrow x_{\star}(t) \quad \text { when } n \rightarrow+\infty, \\
& x^{\prime}\left(t+t_{n}\right) \rightarrow x_{\star}^{\prime}(t) \quad \text { when } n \rightarrow+\infty,
\end{aligned}
$$

uniformly on each compact subinterval of $\mathbb{R}$. By (20) and (21), we deduce that $x \star$ is bounded on $\mathbb{R}$ :

$$
x_{\star}(t) \in K \quad \text { for all } \quad t \in \mathbb{R} .
$$

It remains to prove that $x_{\star}$ is a solution of equation (16). Let $\left(\tau_{1}, \tau_{2}\right)$. Since the function $t \longrightarrow x\left(t+t_{n}\right)$ is a solution of the following equation:

$$
x^{\prime \prime}+\frac{d}{d t}[\nabla F(x)]+G(x)=p^{a p}\left(t+t_{n}\right)+p^{e}\left(t+t_{n}\right),
$$

on $\left(\tau_{1}, \tau_{2}\right)$ and by integrating over $\left(\tau_{1}, t\right)$ for $\tau_{1} \leq t \leq \tau_{2}$, we obtain that $x$ is a solution on $\left(\tau_{1}, \tau_{2}\right)$ of

$$
\begin{aligned}
& x^{\prime}\left(t+t_{n}\right)+\nabla F\left(x\left(t+t_{n}\right)\right)+\int_{\tau_{1}}^{t} G\left(x\left(s+t_{n}\right)\right) d s= \\
& x^{\prime}\left(\tau_{1}+t_{n}\right)+\nabla F\left(x\left(\tau_{1}+t_{n}\right)\right)+\int_{\tau_{1}}^{t} p^{a p}\left(s+t_{n}\right) d s+\int_{\tau_{1}}^{t} p^{e}\left(s+t_{n}\right) d s .
\end{aligned}
$$

Moreover, one has

$$
\int_{\tau_{1}}^{t} p^{e}\left(s+t_{n}\right) d s=\int_{\tau_{1}+t_{n}}^{t+t_{n}} p^{e}(s) d s
$$

and from [Proposition 3.1, [1]], we obtain

$$
\int_{\tau_{1}+t_{n}}^{t+t_{n}} p^{e}(s) d s \rightarrow 0 \quad \text { when } n \rightarrow+\infty .
$$

From (14), (21)-(24), we deduce that $x \star$ satisfies

$$
\chi_{\star}^{\prime}(t)+\nabla F\left(\chi_{\star}(t)\right)+\int_{\tau_{1}}^{t} G\left(\chi_{\star}(s)\right) d s=\chi_{\star}^{\prime}\left(\tau_{1}\right)+\nabla F\left(\chi_{\star}\left(\tau_{1}\right)\right)+\int_{\tau_{1}}^{t} p_{\star}^{a p}(s) d s .
$$

Consequently $\chi \star$ is a solution on $\left(\tau_{1}, \tau_{2}\right)$ for the equation (16). Since this equality is satisfied on every interval $\left(\tau_{1}, \tau_{2}\right)$, then $x_{\star}$ is a solution on $\mathbb{R}$ of equation (16). 
Lemma 11. Let $S$ be a symmetric matrix of order $N$, with eigenvalues $\lambda_{1} \leq \lambda_{2} \leq \ldots \leq \lambda_{N}$. For $R>0$, one has

$$
\min _{(x, y) \in \mathcal{C}}\left(\|y\|^{2}-<S y \mid x>\right)=-\frac{\left(\lambda_{N}-\lambda_{1}\right)^{2}}{16} R^{2}
$$

where

$$
C:=\left\{(x, y) \in \mathbb{R}^{N} \times \mathbb{R}^{N} ; \quad\|x\|=R \quad \text { and } \quad<x \mid y>=0\right\}
$$

Proof. We may assume that $\lambda_{1}=0$, since for $\langle x \mid y\rangle=0$, one has

$$
\|y\|^{2}-<\left(S-\lambda_{1} I\right) y\left|x>=\|y\|^{2}-<S y\right| x>.
$$

We may also assume that $R=1$, since

$$
\|y\|^{2}-<S y\left|R x>=\|y\|^{2}-<R S y\right| x>,
$$

and

$$
\lambda_{\max }(R S)=R \lambda_{\max }(S)=R \lambda_{N} \quad \text { and } \quad \lambda_{\min }(R S)=0,
$$

then the problem (25) is equivalent to

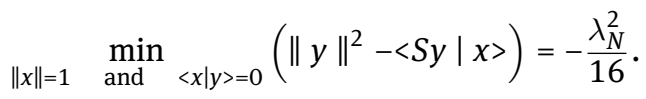

For $\|x\|=1$ and $\langle x| y>=0$, one has

$$
\begin{aligned}
& \left\|\frac{1}{2} S x-\frac{1}{2}<S x \mid x>x-y\right\|^{2} \\
& =\frac{1}{4}\|S x\|^{2}+\frac{1}{4}\langle S x| x>^{2}+\|y\|^{2}-\frac{1}{2}\langle S x \mid x\rangle^{2}-\langle S x \mid y\rangle,
\end{aligned}
$$

then

$$
\begin{aligned}
& \|y\|^{2}-\langle S y \mid x\rangle=\frac{1}{4}\left(\langle S x \mid x\rangle^{2}-\|S x\|^{2}\right) \\
& +\| \frac{1}{2} S x-\frac{1}{2}\langle S x| x>x-y \|^{2} .
\end{aligned}
$$

Due to the fact that $S$ is a symmetric matrix, then there exists an orthonormal basis $\mathcal{B}:=\left\{v_{1}, v_{2}, \ldots, v_{N}\right\}$ of eigenvectors of $S$ with $S v_{i}=\lambda_{i} v_{i}$. With these notations, one has $\|S x\|^{2}=\sum_{i=1}^{N} \lambda_{i}^{2} x_{i}{ }^{2}$ and $\langle S x \mid x\rangle=\sum_{i=1}^{N} \lambda_{i} x_{i}{ }^{2}$ for $x=\sum_{i=1}^{N} x_{i} v_{i}$. Moreover, one has

$$
\sum_{i=1}^{N} \lambda_{i}^{2} x_{i}^{2} \leq 2 \frac{\lambda_{N}}{2} \sum_{i=1}^{N} \lambda_{i} x_{i}^{2} \leq \frac{\lambda_{N}^{2}}{4}+\left(\sum_{i=1}^{N} \lambda_{i} x_{i}^{2}\right)^{2}
$$

then

$$
\langle S x \mid x\rangle^{2}-\|S x\|^{2} \geq-\frac{\lambda_{N}^{2}}{4} .
$$

From (27) and (28), one obtains that

$$
\|y\|^{2}-<S y \mid x>\geq-\frac{\lambda_{N}^{2}}{16},
$$

for all $x$ and $y \in \mathbb{R}^{N}$ such that $\|x\|=1$ and $\langle x| y>=0$. Putting

$$
\bar{x}=\frac{v_{1}+v_{N}}{\sqrt{2}} \quad \text { and } \quad \bar{y}=\frac{1}{2} S \bar{x}-\frac{1}{2}\langle S \bar{x}| \bar{x}>\bar{x} .
$$

one has

$$
\|\bar{x}\|=1 \quad \text { and } \quad\langle\bar{x}| \bar{y}>=0 \text {, }
$$


from which toghether with (27),we deduce

$$
\|\bar{y}\|^{2}-\langle S \bar{y}| \bar{x}>=\frac{1}{4}\left(\langle S \bar{x} \mid \bar{x}\rangle^{2}-\|S \bar{x}\|^{2}\right),
$$

and with $S \bar{x}=\frac{\lambda_{N}}{\sqrt{2}} v_{N}$, we get that

$$
\|\bar{y}\|^{2}-<S \bar{y} \mid \bar{x}>=-\frac{\lambda_{N}^{2}}{16} .
$$

From (29)-(31), one obtains (26), then (25) holds.

\section{Bounded solutions}

In this section, first we study the structure of solutions that are bounded in the future when the second member $\mathrm{p}$ is bounded. Now, we formulate a theorem on the existence of bounded solutions for equation (3).

Definition 12. We say that $-G$ is c-strongly monotone on $\Omega(c>0)$ if

$$
\text { for all } x_{1}, x_{2} \in \Omega, \quad\left\langle G\left(x_{2}\right)-G\left(x_{1}\right)\right| x_{1}-x_{2}>\geq c\left\|x_{1}-x_{2}\right\|^{2} .
$$

Let $\lambda_{M}(x)$ (resp. $\left.\lambda_{m}(x)\right)$ be the greatest (resp. smallest) eigenvalue of the symmetric matrix $\nabla^{2} F(x)$. Denoting $\delta(x):=\lambda_{M}(x)-\lambda_{m}(x)$ (the diameter of the spectrum of $\nabla^{2} F(x)$ ).

Consider the two following hypotheses :

(C1) There exist $x_{0} \in \Omega$ and $R>0$ such that $\bar{B}\left(x_{0}, R\right) \subset \Omega$ and

$$
<p(t)-G\left(x+x_{0}\right) \mid x>\geq \frac{R^{2}}{16} \delta^{2}\left(x+x_{0}\right),
$$

for all $t \in \mathbb{R}$ and $x \in \mathbb{R}^{N}$ such that $\|x\|=R$.

(C2) $\Omega=\mathbb{R}^{N},-G$ is $c$-strongly monotone on $\mathbb{R}^{N}$ and $c>\frac{1}{16} \sup _{x \in \mathbb{R}^{N}} \delta^{2}(x)$.

Theorem 13. Under the hypotheses (H2) and (H3), if hypotheses (C1) or (C2) holds, then equation (3) has at least a bounded solution on $\mathbb{R}$.

Proof. 1) The hypothesis (C2) implies (C1). We choose $x_{0}=0$. For $\|x\|=R$, we have

$$
\langle p(t)-G(x) \mid x\rangle=\langle p(t)-G(0) \mid x\rangle-\langle G(x)-G(0) \mid x\rangle,
$$

and with $-G$ is $c$-strongly monotone, we deduce that

$$
\langle p(t)-G(x)| x>\geq c R^{2}-\left(\|p\|_{\infty}+\|G(0)\|\right) R,
$$

hence for $R$ sufficiently large, we obtain

$$
\langle p(t)-G(x)| x>\geq \frac{R^{2}}{16} \delta^{2}(x),
$$

because

$$
c>\frac{1}{16} \sup _{x \in \mathbb{R}^{N}} \delta^{2}(x) .
$$

2) Assume (C1) is true. Putting $U:=\left\{x-x_{0} ; \quad x \in \Omega\right\}$. $U$ is an open subset of $\mathbb{R}^{N}$ such that $\bar{B}(0, R) \subset U$. Consider

$$
f: \mathbb{R} \times U \times \mathbb{R}^{N} \longrightarrow \mathbb{R}^{N},
$$

defined by

$$
f(t, x, y):=p(t)-G\left(x+x_{0}\right)-\nabla^{2} F\left(x+x_{0}\right) y .
$$


The map $f$ is continuous and equation (3) is equivalent to the following system :

$$
u^{\prime \prime}=f\left(t, u, u^{\prime}\right)
$$

with the change of the unknown function $u(t)=x(t)-x_{0}$. For $t \in \mathbb{R}, x$ and $y \in \mathbb{R}^{N}$ such that $\|x\|=R$ and $<x \mid y>=0$, one has

$$
\begin{aligned}
& \langle f(t, x, y)| x>+\|y\|^{2}=\left\langle p(t)-G\left(x+x_{0}\right) \mid x\right\rangle \\
& +\left(\|y\|^{2}-\left\langle\nabla^{2} F\left(x+x_{0}\right) y\right| x>\right) .
\end{aligned}
$$

By using Lemma 11, we deduce that

$$
\|y\|^{2}-<\nabla^{2} F\left(x+x_{0}\right) y \mid x>\geq-\frac{R^{2}}{16} \delta^{2}\left(x+x_{0}\right),
$$

then

$$
<f(t, x, y) \mid x>+\|y\|^{2} \geq\left\langle p(t)-G\left(x+x_{0}\right)\right| x>-\frac{R^{2}}{16} \delta^{2}\left(x+x_{0}\right) .
$$

Using (C1), one obtains for all $t \in \mathbb{R}, x$ and $y \in \mathbb{R}^{N}$ such that $\|x\|=R$ and $\langle x \mid y\rangle=0$,

$$
<f(t, x, y) \mid x>+\|y\|^{2} \geq 0 .
$$

Moreover, we have the condition for all $t \in \mathbb{R}, x$ and $y \in \mathbb{R}^{N}$ such that $\|x\| \leq R$

$$
\|f(t, x, y)\| \leq \alpha\|y\|+\beta \text {, }
$$

where $\alpha$ and $\beta \in(0,+\infty)$,

$$
\left(\alpha=\sup _{\|x\| \leq R}\left\|\nabla^{2} F\left(x+x_{0}\right)\right\|, \beta=\sup _{\|x\| \leq R}\left\|G\left(x+x_{0}\right)\right\|+\sup _{t \in \mathbb{R}}\|p(t)\|\right) .
$$

By (33) and (34), and from [Theorem 2.3, [8]], we obtain the existence of a solution $u$ of equation (32) which satisfies

$$
\text { for all } t \in \mathbb{R}, \quad\|u(t)\| \leq R,
$$

then equation (3) has a solution $x$ which satisfies

$$
\text { for all } t \in \mathbb{R}, \quad\left\|x(t)-x_{0}\right\| \leq R,
$$

consequently, we obtain the existence of a solution for the equation (3), that is bounded on $\mathbb{R}$.

\section{Particular cases}

\subsection{First particular case: when $F$ is quadratic.}

Now, we consider the following particular case $F(x)=\frac{1}{2}\langle B x \mid x\rangle$ where $B \in \mathcal{L}\left(\mathbb{R}^{N}, \mathbb{R}^{N}\right)$ is symmetric. The equation (3) becomes:

$$
x^{\prime \prime}+B x^{\prime}+G(x)=p(t)
$$

The hypothesis (H2) becomes

(H2-2) $B$ is semi definite positive i.e. : $\langle B x \mid x\rangle \geq 0$ for all $x$ in $\mathbb{R}^{N}$.

Proposition 14. . (i) Any couple of different solutions $x_{1}$ and $x_{2}$ bounded in the future of equation (35), satisfy

$$
<x_{1}(t)-x_{2}(t) \mid x_{1}^{\prime}(t)-x_{2}^{\prime}(t)><0,
$$

for every $t$ where both solutions are defined and

$$
\lim _{t \rightarrow+\infty}\left(\left\|x_{1}(t)-x_{2}(t)\right\|+\left\|x_{1}^{\prime}(t)-x_{2}^{\prime}(t)\right\|\right)=0 .
$$

(ii) Equation (35) has at most one bounded solution on $\mathbb{R}$. 
For the proof, we need the following Lemma :

Lemma 15. Let $p \in B C(\mathbb{R}, X)$. If $x$ is a solution of equation (35), which is bounded on $\mathbb{R}$, then its derivative $x^{\prime}$ is also bounded on $\mathbb{R}$.

Proof. In the particular case where $X$ is a finite-dimensional space, the following estimate

$$
\text { for all } t \in \mathbb{R}, \quad\left\|x^{\prime}(t)\right\| \leq c,
$$

where

$$
c:=\frac{1}{2}\|p\|_{\infty}+\left(\frac{1}{2}\|G\|+2\right)\|x\|_{\infty}+2 \sup _{\|\xi\| \leq\|x\|_{\infty}}\|B(\xi)\|,
$$

is given in (Lemma 3.1, [10]), therefore by continuity of $\nabla F$, the derivative $x^{\prime}$ is bounded on $\mathbb{R}$. However in the infinite-dimensional space, this last estimation on the derivative $x^{\prime}$ and the proof given in [10] are also valid, because this proof use essentially Taylor's formula and integration by parts. By hypothesis $\nabla F$ is Lipschitzian, then $\nabla F$ maps bounded sets into bounded sets, therefore $x^{\prime}$ is bounded on $\mathbb{R}$.

Proof. of Proposition (i) Let $(c,+\infty)$ (with $-\infty \leq c<+\infty)$ be the open interval where both solutions $x_{1}$ and $x_{2}$ are defined. Let $T>c$. Since $x_{1}$ and $x_{2}$ are bounded in the future, there exists a compact $K \subset \Omega$ ( $K$ depends on $T$ ) such that $x_{1}(t)$ and $x_{2}(t) \in K$, for all $t \geq T$. By denoting $h:=x_{1}-x_{2}$, one has

$$
<h^{\prime \prime}(t)\left|h(t)>+<B h^{\prime}(t)\right| h(t)>=<G\left(x_{2}(t)\right)-G\left(x_{1}(t)\right) \mid h(t)>\text {. }
$$

Putting

The function $\alpha$ is derivable and

$$
\alpha(t)=\left\langle h^{\prime}(t)\left|h(t)>+\frac{1}{2}<B h(t)\right| h(t)>\right.
$$

$$
\alpha^{\prime}(t)=<G\left(x_{2}(t)\right)-G\left(x_{1}(t)\right) \mid h(t)>+\left\|h^{\prime}(t)\right\|^{2} .
$$

Since the solutions $x_{1}$ and $x_{2}$ are different, we deduce that $\alpha^{\prime}(t)>0$, for all $t \geq T$, then

$$
\lim _{t \rightarrow+\infty} \alpha(t)=\sup _{t \geq T} \alpha(t)<+\infty .
$$

By the uniform continuity of $\alpha^{\prime}$, one has

$$
\lim _{t \rightarrow+\infty} \alpha^{\prime}(t)=0
$$

then with (36), we deduce that

$$
\lim _{t \rightarrow+\infty}<G\left(x_{2}(t)\right)-G\left(x_{1}(t)\right) \mid h(t)>=0,
$$

and

$$
\lim _{t \rightarrow+\infty}\left\|h^{\prime}(t)\right\|=0
$$

From (38) and Lemma 7 , we obtain

$$
\lim _{t \rightarrow+\infty}\|h(t)\|=0 \text {. }
$$

With (39) and (40) we deduce that $\lim _{t \rightarrow+\infty} \alpha(t)=0$, then with (37), we obtain $\alpha(t)<0$ for all $t \geq T$, since $\alpha$ is strictly monotone. From $\mathbf{H 2}$ - 2), we deduce

$$
\text { for all } t \geq T, \quad<h^{\prime}(t) \mid h(t)><0 .
$$

The equation (41) is true for all $T>c$, then

$$
\text { for all } t>c, \quad<h^{\prime}(t) \mid h(t)><0 .
$$

From (39), (40) and (42), we have the following result: with the same computations, one has if $x_{1}$ and $x_{2}$ are two different bounded solutions on $\mathbb{R}, \lim _{t \rightarrow-\infty}\|h(t)\|=0$ which is a contradiction since $t \rightarrow\|h(t)\|$ is nonnegative and strictly decreasing. 
Theorem 16. Assume that the forcing term $p$ is almost periodic.

(i) If equation (35) has at least one solution that is bounded in the future, then equation (35) has exactly one solution $x$ that is bounded on $\mathbb{R}$. Moreover this solution $x$ and its derivatives $x^{\prime}$ and $x^{\prime \prime}$ are almost periodic and satisfy $\bmod (x) \subset \bmod (p)$.

(ii) Every y bounded solution in the future is asymptotically almost periodic

$$
\lim _{t \rightarrow+\infty}\left(\|x(t)-y(t)\|+\left\|x^{\prime}(t)-y^{\prime}(t)\right\|\right)=0 .
$$

Proof. i) Let $\phi$ be a solution of equation (35) which is bounded in the future. Then there exist a compact $K \subset \Omega$ and $t_{0} \in \mathbb{R}$ such that

$$
\text { for all } t \geq t_{0}, \quad \phi(t) \in K .
$$

By Lemma 9, there exists $c_{1}>0$ such that $\sup _{t \geq t_{0}}\left\|\phi^{\prime}(t)\right\| \leq c_{1}$. If we denote by $K_{0}:=K \times \bar{B}\left(0, c_{1}\right)$, the compact subset included in $\Omega \times \mathbb{R}^{N}$, then we have

$$
\bigcup_{t \geq t_{0}}\left\{\left(\phi(t), \phi^{\prime}(t)\right)\right\} \subset K_{0} .
$$

By [Theorem 6.2, p. 99, [14]], equation (35) has at least one solution $x$ defined on $\mathbb{R}$ such that

$$
\bigcup_{t \in \mathbb{R}}\left\{\left(x(t), x^{\prime}(t)\right)\right\} \subset K_{0},
$$

then $x$ is bounded on $\mathbb{R}$. By Proposition 14, we have the uniqueness of solution that is bounded on $\mathbb{R}$. For each $p_{\star} \in H(p)$ (the hull set of $p$ ), we consider the following equation

$$
x^{\prime \prime}+B x^{\prime}+G(x)=p_{\star}(t) .
$$

By (44) and from [Lemma 4.3, p. 104, [11]], there exists a solution $x \star$ of equation (45) such that

$$
\bigcup_{t \in \mathbb{R}}\left\{\left(x_{\star}(t), x_{\star}^{\prime}(t)\right)\right\} \subset K_{0}
$$

By proposition 14, for each $p_{\star} \in H(p)$, equation (45) has exactly one solution $x_{\star}$ satisfying (46). By using [Theorem 10, p. 170, [14]], we obtain the bounded solution $x$; so that $x$ and its derivative $x^{\prime}$ are almost periodic and $\bmod (x) \subset \bmod (p)$. The almost periodicity of the second derivative is a direct consequence of equation (35).

(ii) Since the almost periodic solution $x$ is bounded in the future, by Proposition 14, we obtain (43).

Theorem 17. Let $c \in \mathbb{R}$. Assume that $p$ in equation (35) is the sum $p=p^{a p}+p^{e}$, where $p^{a p} \in A P\left(\mathbb{R}, \mathbb{R}^{N}\right)$ and $p^{e} \in \mathcal{E}^{+}\left((c,+\infty) ; \mathbb{R}^{N}\right)$.

(i) If equation (35) has at least one solution that is bounded in the future, then equation

$$
x^{\prime \prime}+B x^{\prime}+G(x)=p^{a p}(t)
$$

has exactly one solution $\phi$ that is bounded on $\mathbb{R}$. Moreover, this solution $\phi$ and its derivatives $\phi^{\prime}$ and $\phi^{\prime \prime}$ are almost periodic and $\bmod (\phi) \subset \bmod \left(p^{a p}\right)$.

(ii) Every solution $x$ that is bounded in the future of equation (35) is asymptotically almost periodic. i.e.:

$$
\lim _{t \rightarrow+\infty}\left(\|x(t)-\phi(t)\|+\left\|x^{\prime}(t)-\phi^{\prime}(t)\right\|\right)=0
$$

Proof. (i) From Theorem 16, it suffices to prove that equation (47) has at least one solution which is bounded in the future. Let $x$ be a solution that is bounded in the future of equation (35). Since $p^{a p}$ is almost periodic, there exists a numerical sequence $\left(t_{n}\right)_{n}$ such that

$$
\lim _{n \rightarrow+\infty} t_{n}=+\infty \text { and } \lim _{n \rightarrow+\infty} \sup _{t \in \mathbb{R}}\left\|p^{a p}\left(t+t_{n}\right)-p^{a p}(t)\right\|=0 .
$$


By Lemma 10, we deduce the existence of a bounded solution $\phi$ on $\mathbb{R}$ for the equation (47). The Theorem 16 , gives us the uniqueness of bounded solution $\phi$ on $\mathbb{R}$, the almost periodicity of $\phi, \phi^{\prime}$ and $\phi^{\prime \prime}$; and the formula of modules.

(ii) We claim that

$$
\lim _{t \rightarrow-\infty}\|x(t)-\phi(t)\|=0 .
$$

Assume the contrary, there exist $\varepsilon>0$ and a numerical sequence $\left(t_{n}\right)_{n}$ satisfying

$$
\lim _{n \rightarrow+\infty} t_{n}=-\infty \text { and } \inf _{n \in \mathbb{N}}\left\|x\left(t_{n}\right)-\phi\left(t_{n}\right)\right\| \geq \varepsilon .
$$

Since $p^{a p}$ is almost periodic, there exist a subsequence of $\left(t_{n}\right)_{n}$ and $p_{\star}^{a p} \in A P\left(\mathbb{R}, \mathbb{R}^{N}\right)$ satisfying (14). By Lemma 10, we deduce the existence of a subsequence of $\left(t_{n}\right)_{n}$ and the existence of solution $X \star$ bounded on $\mathbb{R}$ for the equation

$$
\chi_{\star}^{\prime \prime}+B x_{\star}^{\prime}+G\left(x_{\star}\right)=p_{\star}^{a p}(t) ;
$$

satisfying (15). Since $\phi$ is an almost periodic solution of equation (47), then $\phi$ is a bounded solution on $\mathbb{R}$ for the equation (35) with the perturbation $p^{e}=0$. By Lemma 10 and (14), we obtain the existence of a subsequence of $\left(t_{n}\right)_{n}$ such that

$$
\text { for all } t \in \mathbb{R}, \quad \lim _{n \rightarrow+\infty} \phi\left(t+t_{n}\right)=\phi_{\star}(t),
$$

where $\phi_{\star}$ is a bounded solution on $\mathbb{R}$ of equation (51). By (15), (50) and (52), we deduce that

$$
\left\|\chi_{\star}(0)-\phi_{\star}(0)\right\| \geq \varepsilon \text {. }
$$

Since $x_{\star}$ and $\phi_{\star}$ are two bounded solutions on $\mathbb{R}$ of equation (51), we obtain assertion $\mathbf{i}$ ) of this theorem, which gives the equality of the last two solutions, which contradicts (53), consequently (49) is satisfied. Since the function $x^{\prime \prime}-\phi^{\prime \prime}$ is bounded on $\left(t_{0},+\infty\right)$ (c.f. Lemma 7$)$, then $x^{\prime}-\phi^{\prime}$ is uniformly continuous on $\left(t_{0},+\infty\right)$, then (49) implies that

$$
\lim _{t \rightarrow-\infty}\left\|x^{\prime}(t)-\phi^{\prime}(t)\right\|=0
$$

consequently (ii) is proved.

Denoting $\mathcal{E}\left(\mathbb{R} ; \mathbb{R}^{N}\right)$ the set defined by $p \in \mathcal{E}\left(\mathbb{R}, \mathbb{R}^{N}\right)$ if and only if $p \in C_{b}\left(\mathbb{R} ; \mathbb{R}^{N}\right)$ and

$$
\lim _{n \rightarrow-\infty} \int_{n}^{n+1}\|p(t)\| d t=0 .
$$

Proposition 18. Suppose that $p=p^{a p}+p^{e}$ where $p^{a p} \in A P\left(\mathbb{R}, \mathbb{R}^{N}\right)$ and $p^{e} \in \mathcal{E}\left(\mathbb{R}, \mathbb{R}^{N}\right)$. If equation (35) has a bounded solution $x$ on $\mathbb{R}$, then this solution is unique and asymptotically almost periodic at $-\infty$ and at $+\infty$ :

$$
\lim _{|t| \rightarrow+\infty}\left(\|x(t)-\phi(t)\|+\left\|x^{\prime}(t)-\phi^{\prime}(t)\right\|\right)=0
$$

where $\phi$ denotes the almost periodic solution of equation (47).

Proof. Uniqueness of bounded solution on $\mathbb{R}$ of equation (35) derives from Proposition 14. By using Theorem 17 , we obtain the existence of the almost periodic solution of equation (47) satisfying (48). The proof of

$$
\lim _{t \rightarrow-\infty}\left(\|x(t)-\phi(t)\|+\left\|x^{\prime}(t)-\phi^{\prime}(t)\right\|\right)=0,
$$

is similar to the one of (48). So (55) is proved. 


\subsection{Second particular case : $G$ is linear}

Now we consider the particular case $G(x)=C x$ with $C \in \mathcal{L}\left(\mathbb{R}^{N}, \mathbb{R}^{N}\right)$. The equation (3) becomes:

$$
x^{\prime \prime}+\frac{d}{d t}[\nabla F(x)]+C x=p(t)
$$

The hypothesis (H1) becomes (H1-3) $C$ is a definite negative and symmetric matrix : $\langle C x \mid x\rangle\langle 0$ for all $x$ in $\mathbb{R}^{N}$ such that $x \neq 0$.

Proposition 19. (i) If $x_{1}$ and $x_{2}$ are different solutions bounded in the future for equation (56), then

$$
\lim _{t \rightarrow+\infty}\left\|x_{1}(t)-x_{2}(t)\right\|+\left\|x_{1}^{\prime}(t)-x_{2}^{\prime}(t)\right\|=0 .
$$

(ii) Equation (56) has an unique bounded solution on $\mathbb{R}$.

Proof. (i) Let $(c,+\infty)$ (with $-\infty \leq c<+\infty$ ) be an open subset where both solutions $x_{1}$ and $x_{2}$ are defined. Since $x_{1}$ and $x_{2}$ are bounded in the future, then there exist a compact $K \subset \Omega$ and $T_{0}>c$ such that $x_{1}(t)$ and $x_{2}(t) \in K$ for all $t \geq T_{0}$. Denoting $h:=x_{1}-x_{2}$ and $k(t):=\nabla F\left(x_{1}(t)\right)-\nabla F\left(x_{2}(t)\right)$, one has

$$
h^{\prime \prime}+k^{\prime}+C h=0 .
$$

Using Lemma 9 and (58), we deduce that $h^{\prime}, h^{\prime \prime}, k$ and $k^{\prime}$ are bounded on $\left(T_{0},+\infty\right)\left(\sup _{t>T_{0}}\left|h^{\prime}(t)\right|<+\infty\right)$.

Putting

$$
f(t):=\frac{1}{2}<C^{-1}\left(h^{\prime}(t)+k(t)\right) \mid h^{\prime}(t)+k(t)>+\frac{1}{2}\|h(t)\|^{2} .
$$

The function $f$ is bounded and continuously differentiable on $\left(T_{0},+\infty\right)$, moreover one has

$$
f^{\prime}(t)=-<k(t) \mid h(t)>\text {. }
$$

From the convexity of $F$, we deduce that $f^{\prime}(t) \leq 0$, then

$$
\lim _{t \rightarrow+\infty} f(t)=\inf _{t>T_{0}} f(t) \in \mathbb{R} .
$$

The second derivative of $f$ is bounded, then $f^{\prime}$ is uniformly continuous, so with (60), we deduce that $\lim _{t \rightarrow+\infty} f^{\prime}(t)=0$, then with (59), we obtain

$$
\lim _{t \rightarrow+\infty}<k(t) \mid h(t)>=0 .
$$

From Lemma 4 we deduce that

$$
\lim _{t \rightarrow+\infty}\|k(t)\|=0 .
$$

We will establish (57). Even if it means to work on the eigenspaces of $C$, we can assume that $C=\lambda I$, with $\lambda<0$. Let $\lambda=-\omega^{2}$, we can assume that $\lambda=-1$, even if it means to replace $h$ and $k$ by $h_{1}(t)=h\left(\frac{t}{\omega}\right)$ and $k_{1}(t)=\frac{1}{\omega} k\left(\frac{t}{\omega}\right)$. Then, one has

$$
h^{\prime \prime}+k^{\prime}-h=0 .
$$

By the variation constant formula, we obtain that the solutions $h$ which are bounded in the future of equation (63) are given by

$$
h(t)=\frac{1}{2} e^{-t} \int_{0}^{t} k^{\prime}(s) e^{s} d s+\frac{1}{2} e^{t} \int_{t}^{+\infty} k^{\prime}(s) e^{-s} d s+c e^{-t} .
$$

We can assume that $c=0$, due to the fact that $t \longrightarrow e^{-t}$ goes to zero, so is for its derivative when $t$ tends to $+\infty$. By integration by parts, we obtain

$$
e^{-t} \int_{0}^{t} k^{\prime}(s) e^{s} d s=k(t)-k(0) e^{-t}-e^{-t} \int_{0}^{t} k(s) e^{s} d s .
$$


By (62) and by making cut out of the integral, we deduce that

$$
\lim _{t \rightarrow+\infty} e^{-t} \int_{0}^{t} k(s) e^{s} d s=0 .
$$

Similarly, we have

$$
\begin{aligned}
& e^{t} \int_{t}^{+\infty} k^{\prime}(s) e^{-s} d s=e^{t}\left[k(s) e^{-s}\right]_{t}^{+\infty}+e^{t} \int_{t}^{+\infty} k(s) e^{-s} d s \\
& =-k(t)+e^{t} \int_{t}^{+\infty} k(s) e^{-s} d s,
\end{aligned}
$$

It results that

$$
\lim _{t \rightarrow+\infty}\|h(t)\|=0
$$

Since $h^{\prime}$ is uniformly continuous, we deduce that

$$
\lim _{t \rightarrow+\infty}\left\|h^{\prime}(t)\right\|=0,
$$

then, the proof of (57) is finished.

(ii) Let $x_{1}$ and $x_{2}$, be 2 bounded and different solutions defined on $\mathbb{R}$. From (57), (60) and (62), we obtain that

$$
\lim _{t \rightarrow+\infty} f(t)=\inf _{t \in \mathbb{R}} f(t)=0 .
$$

With the same argument, we deduce that

$$
\lim _{t \rightarrow-\infty} f(t)=\sup _{t \in \mathbb{R}} f(t)=0,
$$

Since $f$ is decreasing, we obtain $f(t)=0$, it follows that $f^{\prime}(t)=0$ for all $t \in \mathbb{R}$. By using Lemma 3 and (59), one has $k(t)=0$ for all $t \in \mathbb{R}$ and with (58), we deduce that

$$
\frac{d^{2}}{d t^{2}}\left(\frac{1}{2}\|h(t)\|^{2}\right)=-<C h(t) \mid h(t)>+\left\|h^{\prime}\right\|^{2} .
$$

Putting $r(t):=\frac{1}{2}\|h(t)\|^{2}$. Since $C$ is negative definite, we have $r^{\prime \prime}(t) \geq 0$ for all $t \in \mathbb{R}$, then $r$ is convex and bounded in $\mathbb{R}$, consequently $r$ is constant on $\mathbb{R}$, consequently $r^{\prime \prime}(t)=0$ for all $t \in \mathbb{R}$. From hypothesis madded on $C$ and by (64), we deduce that $h(t)=0$, then $x_{1}(t)=x_{2}(t)$ for all $t \in \mathbb{R}$.

Theorem 20. Assume $p$ is almost periodic

(i) If equation (56) has at least one solution that is bounded in the future, then (56) has exactly one bounded solution on $\mathbb{R}$. Moreover, this solution $x$ and its derivatives $x^{\prime}$ and $x^{\prime \prime}$ are almost periodic and $\bmod (x) \subset \bmod (p)$.

(ii) Every bounded solution in the future $y$ is asymptotically almost periodic:

$$
\lim _{t \rightarrow+\infty}\left(\|x(t)-y(t)\|+\left\|x^{\prime}(t)-y^{\prime}(t)\right\|\right)=0 .
$$

Proof. (i) The proof is similar to the one given in Theorem 16. Let $\phi$ be a solution that is bounded in the future of equation (56). There exist a compact $K \subset \Omega$ and $t_{0} \in \mathbb{R}$ such that

$$
\text { for all } t \geq t_{0}, \quad \phi(t) \in K .
$$

By Lemma 9, there exists $c_{1}>0$ such that $\sup _{t \geq t_{0}}\left\|\phi^{\prime}(t)\right\| \leq c_{1}$. If we denote $K_{0}:=K \times \bar{B}\left(0, c_{1}\right)$ the compact subset of $\Omega \times \mathbb{R}^{N}$, we obtain

$$
\bigcup_{t \geq t_{0}}\left\{\left(\phi(t), \phi^{\prime}(t)\right)\right\} \subset K_{0} .
$$


From [Theorem 6.2, p. 99, [14]] and [Lemma 4.3, p. 104, [4]], we obtain that for each $p_{\star} \in H(p)$, there exists a solution $x \star$ of equation

$$
x^{\prime \prime}+\frac{d}{d t}[\nabla F(x)]+C x=p_{\star}(t)
$$

such that

$$
\bigcup_{t \in \mathbb{R}}\left\{\left(x_{\star}(t), x_{\star}^{\prime}(t)\right)\right\} \subset K_{0}
$$

By proposition 19, for each $p_{\star} \in H(p)$, equation (66) has an unique solution $x \star$ satisfying (59). By using [Theorem 10, p. 170, [14]], we obtain that the bounded solution $x$; so that $x$ and its derivative $x^{\prime}$ are almost periodic and $\bmod (x) \subset \bmod (p)$. The almost periodicity of the second derivative $x$ " follows from (56).

(ii) Let us prove that

$$
\lim _{t \rightarrow-\infty}\|x(t)-y(t)\|=0 .
$$

Assume the contrary, then there exist $\varepsilon>0$ and a numerical sequence $\left(t_{n}\right)_{n}$ satisfying

$$
\lim _{n \rightarrow+\infty} t_{n}=-\infty \text { and } \inf _{n \in \mathbb{N}}\left\|x\left(t_{n}\right)-y\left(t_{n}\right)\right\| \geq \varepsilon
$$

Since $p$ is almost periodic, there exist a subsequence of $\left(t_{n}\right)_{n}$ and $p_{\star} \in A P\left(\mathbb{R}, \mathbb{R}^{N}\right)$ verifying

$$
\lim _{n \rightarrow+\infty} \sup _{t \in \mathbb{R}}\left\|p\left(t+t_{n}\right)-p_{\star}(t)\right\|=0 \text {. }
$$

From Lemma 10 we deduce (The ergodic perturbation of $p$ is zero : $p^{e}=0$ ), Existence of a subsequence of $\left(t_{n}\right)_{n}$ and existence of a solution $x_{\star}\left(\right.$ resp. $y_{\star}$ ) bounded on $\mathbb{R}$ of equation (66) verifying

$$
\begin{aligned}
& \text { for all } t \in \mathbb{R}, \quad \lim _{n \rightarrow+\infty} x\left(t+t_{n}\right)=x_{\star}(t), \\
& \text { for all } t \in \mathbb{R}, \quad \lim _{n \rightarrow+\infty} y\left(t+t_{n}\right)=y_{\star}(t) .
\end{aligned}
$$

From (69)-(71), we deduce that

$$
\|x \star(0)-y \star(0)\| \geq \epsilon \text {. }
$$

Since $x \star$ and $y \star$ are two bounded solutions on $\mathbb{R}$ of equation (66), From the assertion i) of this theorem, we obtain that, the equality of these last solutions, which is a contradiction with (72), consequently (68) is satisfied. Since the function $x^{\prime \prime}-y^{\prime \prime}$ is bounded on $\left(t_{0},+\infty\right)$ (c.f. Lemma 9), then $x^{\prime}-y^{\prime}$ is uniformly continuous on $\left(t_{0},+\infty\right)$, then (68) implies that

$$
\lim _{t \rightarrow-\infty}\left\|x^{\prime}(t)-y^{\prime}(t)\right\|=0
$$

which ends the proof of ii).

Theorem 21. Suppose that $p=p^{a p}+p^{e}$ where $p^{a p} \in A P\left(\mathbb{R}, \mathbb{R}^{N}\right)$ and $p^{e} \in \mathcal{E}^{+}\left((c,+\infty), \mathbb{R}^{N}\right)$. i) If equation (56) has at least one solution that is bounded in the future, then equation

$$
x^{\prime \prime}+\frac{d}{d t}[\nabla F(x)]+C x=p^{a p}(t)
$$

has a unique solution $\phi$ that is bounded on $\mathbb{R}$. Moreover, this solution $\phi$ and its derivatives $\phi^{\prime}$ and $\phi^{\prime \prime}$ are almost periodic and $\bmod (\phi) \subset \bmod \left(p^{a p}\right)$.

ii) Every solution $x$ bounded in the future of equation (56) is asymptotically almost periodic:

$$
\lim _{t \rightarrow+\infty}\left(\|x(t)-\phi(t)\|+\left\|x^{\prime}(t)-\phi^{\prime}(t)\right\|\right)=0
$$

Proof. The proof is similar to the one given in Theorem 17

(i) From Theorem 20, it suffices to prove that equation (73) has at least one solution bounded in the future. 
Let $x$ be a solution that is bounded in the future for the equation (56). Since $p^{a p}$ is almost periodic, then there exists a numerical sequence $\left(t_{n}\right)_{n}$ satisfying

$$
\lim _{n \rightarrow+\infty} t_{n}=+\infty \text { and } \lim _{n \rightarrow+\infty} \sup _{t \in \mathbb{R}}\left\|p^{a p}\left(t+t_{n}\right)-p^{a p}(t)\right\|=0 .
$$

From Lemma 10, we deduce the existence of a solution $\phi$ that is bounded on $\mathbb{R}$ for the equation (73). By Theorem 20, we deduce the uniqueness of the bounded solution $\phi$ on $\mathbb{R}$, and that $\phi, \phi^{\prime}$ and $\phi^{\prime \prime}$ are almost periodic and the formula of the module.

(ii) We claim that

$$
\lim _{t \rightarrow-\infty}\|x(t)-\phi(t)\|=0 .
$$

Assume the contrary, then there exist $\varepsilon>0$ and a numerical sequence $\left(t_{n}\right)_{n}$ verifying

$$
\lim _{n \rightarrow+\infty} t_{n}=+\infty \text { and } \inf _{n \in \mathbb{N}}\left\|x\left(t_{n}\right)-\phi\left(t_{n}\right)\right\| \geq \epsilon .
$$

Since $p^{a p}$ is an almost periodic function, then there exist a subsequence of $\left(t_{n}\right)_{n}$ and $p_{\star}^{a p} \in A P\left(\mathbb{R}, \mathbb{R}^{N}\right)$ verifying (14). From Lemma 10, we deduce the existence of a subsequence of $\left(t_{n}\right)_{n}$ and the existence of a $x_{\star}$ bounded on $\mathbb{R}$ of the following equation

$$
x_{\star}^{\prime \prime}+\frac{d}{d t}\left[\nabla F\left(x_{\star}\right)\right]+C x_{\star}=p_{\star}^{a p}(t) .
$$

satisfying (15). Since $\phi$ is an almost periodic solution of equation (73), then $\phi$ is a bounded solution on $\mathbb{R}$ of equation (56) with the perturbation null: $p^{e}=0$. By Lemma 10 and by (14), there exists a subsequence of $\left(t_{n}\right)_{n}$ such that

$$
\text { for all } t \in \mathbb{R}, \quad \lim _{n \rightarrow+\infty} \phi\left(t+t_{n}\right)=\phi_{\star}(t),
$$

where $\phi_{\star}$ is a bounded solution on $\mathbb{R}$ of equation (76). From (15), (??) and (77), one has

$$
\left\|\chi_{*}(0)-\phi_{\star}(0)\right\| \geq \varepsilon .
$$

Since $x_{\star}$ and $\phi_{\star}$ are two bounded solutions on $\mathbb{R}$ of equation (76), By assertion i) of this theorem, we obtain $x_{\star}(t)=\phi_{\star}(t)$ for each $t \in \mathbb{R}$, which contradicts (78), Therefore (75) is satisfied. Since $x^{\prime \prime}-\phi^{\prime \prime}$ is bounded on $\left(t_{0},+\infty\right)$ (c.f. Lemma 9), then $x^{\prime}-\phi^{\prime}$ is uniformly continuous on $\left(t_{0},+\infty\right)$, hence (75) implies

$$
\lim _{t \rightarrow-\infty}\left\|x^{\prime}(t)-\phi^{\prime}(t)\right\|=0,
$$

which ends the proof of (ii).

Proposition 22. Assume that $p=p^{a p}+p^{e}$ where $p^{a p} \in A P\left(\mathbb{R}, \mathbb{R}^{N}\right)$ and $p^{e} \in \mathcal{E}\left(\mathbb{R} ; \mathbb{R}^{N}\right)$. If equation (56) has a solution $x$ that is bounded on $\mathbb{R}$, then this solution is unique and is asymptotically almost periodic at $-\infty$ and at $+\infty$ :

$$
\lim _{|t| \rightarrow+\infty}\left(\|x(t)-\phi(t)\|+\left\|x^{\prime}(t)-\phi^{\prime}(t)\right\|\right)=0
$$

where $\phi$ denotes the almost periodic solution of equation (73).

Proof. The uniqueness of the bounded solution of equation 56 results from proposition 19 . By using Theorem 21 , we obtain the existence of the almost periodic solution of equation (73) verifying (74). The proof of

$$
\lim _{t \rightarrow-\infty}\left(\|x(t)-\phi(t)\|+\left\|x^{\prime}(t)-\phi^{\prime}(t)\right\|\right)=0,
$$

is similar to the proof of (74). Then the equality (79) is achieved.

Now, we state a result of existence and uniqueness of the $\mu$-pseudo almost periodic solution. 


\section{$5 \mu$ - Pseudo almost periodic functions}

In this section, we define new concepts, the $\mu$-ergodic functions and the $\mu$-pseudo almost periodic functions, then we give some properties of these functions. The notion of $\mu$-pseudo almost periodicity is a generalization of the pseudo almost periodicity introduced by Zhang [20]; it is also a generalization of weighted pseudo almost periodicity given by Diagana [12]. For more details on this functions we refer the reader to [13].

Throughout this section $E$ is a Banach space and $B C(\mathbb{R}, E)$ denotes the Banach space of bounded continuous functions from $\mathbb{R}$ to $E$, equipped with the supremum norm $\|f\|_{\infty}=\sup _{t \in \mathbb{R}}\|f(t)\|$. We denote by $\boldsymbol{B}$ the Lebesgue $\sigma$ - field of $\mathbb{R}$ and by $\mathcal{M}$ the set of all positive measures $\mu$ on $\boldsymbol{B}$ satisfying $\mu(\mathbb{R})=+\infty$ and $\mu([a, b])<+\infty$ for all $a, b \in \mathbb{R}(a \leq b)$.

From $\mu \in \mathcal{M}$, we formulate the following hypotheses:

$\left(\mathbf{H}_{1}\right)$ For all $a, b$ and $c \in \mathbb{R}$, such that $0 \leq a<b \leq c$, there exist $\tau_{0} \geq 0$ and $\alpha_{0}>0$ such that

$$
|\tau| \geq \tau_{0} \Rightarrow \mu((a+\tau, b+\tau)) \geq \alpha_{0} \mu((\tau, c+\tau)) .
$$

$\left(\mathbf{H}_{2}\right)$ For all $\tau \in \mathbb{R}$, there exist $\beta>0$ and a bounded interval $I$ such that $\mu(\{a+\tau, a \in A\}) \leq \beta \mu(A)$, when $A \in \boldsymbol{B}$ satisfies $A \cap I=\emptyset$.

Definition 23. Let $\mu \in \mathcal{M}$. A bounded continuous function $f: \mathbb{R} \rightarrow E$ is said to be $\mu$ - ergodic if

$$
\lim _{r \rightarrow+\infty} \frac{1}{\mu([-r, r])} \int_{[-r, r]}\|f(t)\| d \mu(t)=0 .
$$

We denote the space of all such functions by $\mathcal{E}(\mathbb{R}, \mathbb{E}, \mu)$.

Definition 24. Let $\mu \in \mathcal{M}$. A bounded continuous function $f: \mathbb{R} \rightarrow \mathbb{E}$ is said to be $\mu$-pseudo almost periodic if $f$ is written in the form:

$$
f=g+\varphi,
$$

where $g \in A P(\mathbb{R}, \mathbb{E})$ and $\varphi \in \mathcal{E}(\mathbb{R}, \mathbb{E}, \mu)$.

We denote the space of all such functions by $\operatorname{PAP}(\mathbb{R}, \mathbb{E}, \mu)$, then we have the following inclusions

$$
A P(\mathbb{R}, E) \subset P A P(\mathbb{R}, \mathbb{E}, \mu) \subset B C(\mathbb{R}, E) .
$$

Proposition 25. [13] Let $\mu \in \mathcal{N}$. Then $\mathcal{E}\left(\mathbb{R}, \mathbb{E}, \mu,\|\cdot\|_{\infty}\right)$ is a Banach space.

From the definition of $P A P(\mathbb{R}, \mathbb{E}, \mu)$, we easily deduce the following result:

Proposition 26. [13] Let $\mu \in \mathcal{M}$. Then $\operatorname{PAP}(\mathbb{R}, \mathbb{E}, \mu)$ is a vector space.

Next result is a characterization of $\mu$ - ergodic functions.

Theorem 27. [13] Let $\mu \in \mathcal{M}$ and I be a bounded interval (eventually $I=\emptyset$ ). Assume that $f \in B C(\mathbb{R}, E)$. The following assertions are equivalent:

i) $f \in \mathcal{E}(\mathbb{R}, \mathbb{E}, \mu)$.

ii) $\lim _{r \rightarrow+\infty} \frac{1}{\mu([-r, r] \backslash I)} \int_{[-r, r] \backslash I}\|f(t)\| d \mu(t)=0$.

iii) For any $\varepsilon>0, \lim _{r \rightarrow+\infty} \frac{\mu(\{t \in([-r, r] \backslash I):\|f(t)\|>\varepsilon\})}{\mu([-r, r] \backslash I)}=0$.

Corollary 28. [13] Let $\mu \in \mathcal{M}$ satisfy $\left(\boldsymbol{H}_{1}\right)$. Then the decomposition of a $\mu$-pseudo almost periodic function in the form $f=g+\varphi$, where $g \in A P(\mathbb{R}, \mathbb{E})$ and $\varphi \in \mathcal{E}(\mathbb{R}, \mathbb{E}, \mu)$, is unique. 
Corollary 29. Let $\mu \in \mathcal{M}$ satisfy $\left(\boldsymbol{H}_{1}\right)$. Then $P A P\left(\mathbb{R}, \mathbb{E}, \mu,\|\cdot\|_{\infty}\right)$ is a Banach space.

Denoting $\lambda_{M}(x)$ (resp. $\lambda_{m}(x)$ ) the greatest (resp. smallest) eigenvalues of the symmetric matrix $\nabla^{2} F(x)$. Putting $\delta(x):=\lambda_{M}(x)-\lambda_{m}(x)$ (The spectrum diameter of $\nabla^{2} F(x)$ ). Since $C$ is negative definite and symmetric, there exists $c>0$ such that $\langle C x \mid x\rangle \leq-c\|x\|^{2}$ for all $x$ in $\mathbb{R}^{N}$.

Consider the following hypotheses:

(F1) There exist $x_{0} \in \Omega$ and $R>0$ such that $\bar{B}\left(x_{0}, R\right) \subset \Omega$ and

$$
<p(t)-C x_{0} \mid x>\geq R^{2}\left(\frac{1}{16} \delta^{2}(x)-c\right),
$$

for all $t \in \mathbb{R}$ and $x \in \mathbb{R}^{N}$ with $\|x\|=R$.

(F2) $\Omega=\mathbb{R}^{N}$ and $c>\frac{1}{16} \sup _{x \in \mathbb{R}^{N}} \delta^{2}(x)$.

Corollary 30. Assume that (F1) or (F2) is true, then equation (56) has an unique bounded solution on $\mathbb{R}$.

Proof. It is a direct consequence of Theorem 13, applied with $G(x)=C x$ and the Proposition 19.

Theorem 31. Suppose that $p$ is $\mu$-pseudo almost periodic.

(i) If hypothesis (F1) or (F2) is verified, then equation (56) has an unique solution $x$ that is bounded on $\mathbb{R}$ which is $\mu$-pseudo almost periodic and verifies $\bmod (x) \subset \bmod (p)$.

(ii) Moreover, if we denote by $p^{a p}$ (resp. $y$ ) the almost periodic component of $p$ (resp. $x$ ), then $y$ is the almost periodic solution of (73).

Remark 32. In the almost periodic case $\left(p \in A P\left(\mathbb{R}, \mathbb{R}^{N}\right)\right)$, The unique bounded solution is almost periodic and verifies the module's formula.

Proof. The proof is similar to the one given in Theorem 34.

From Corollary 30, one obtains the existence and uniqueness of the solution $x$ that is bounded on $\mathbb{R}$ for the equation (56).

We state the existence and uniqueness of the almost periodic solution $y$ of equation (73) and $\bmod (y) \subset$ $\bmod \left(p^{a p}\right)$. If $p$ verifies $(\mathbf{F} 1)$ or $(\mathbf{F} 2)$, then $p^{a p}$ verifies one of the two hypotheses. In fact for (F2), it is evident since (F2) depends only of $p$. For (F1), it suffices to make the following remark, if one has

$$
<p(t)-C x_{0} \mid x>\geq R^{2}\left(\frac{1}{16} \delta^{2}(x)-c\right),
$$

then, we have

$$
<p^{a p}(t)-C x_{0} \mid x>\geq R^{2}\left(\frac{1}{16} \delta^{2}(x)-c\right),
$$

Since $t \longrightarrow\left\langle p^{a p}(t)-C x_{0} \mid x\right\rangle$ is the almost periodic component of $t \longrightarrow\left\langle p(t)-C x_{0} \mid x\right\rangle$. Follows Corollary 30, equation (73) has an unique solution $y$ which is bounded on $\mathbb{R}$. By using Theorem 20, the bounded solution $y$ is almost periodic and satisfies the formula of modules.

Putting $h:=x-y$. To end the proof, it suffices to prove that $h \in P A P_{0}\left(\mathbb{R}, \mathbb{R}^{N}\right)$. Since $x($ resp. $y$ ) is a solution of (56) (resp. (73)), then $h$ verifies

$$
h^{\prime \prime}+k^{\prime}+C k=p^{e}(t)
$$

where $p^{e}$ denotes the ergodic perturbation of $p$ and $k(t):=\nabla F(x(t))-\nabla F(y(t))$. Putting

$$
f(t):=\frac{1}{2}<C^{-1}\left(h^{\prime}(t)+k(t)\right) \mid h^{\prime}(t)+k(t)>+\frac{1}{2}\|h(t)\|^{2} .
$$

The function $f$ is derivable and

$$
f^{\prime}(t)=-<k(t), h(t)>+<C^{-1} p^{e}(t) \mid h^{\prime}(t)+k(t)>,
$$


with the convexity of $F$ and by (82), we have

$$
0 \leq<k(t) \mid h(t)>\leq\left\|C^{-1}\left(h^{\prime}+k\right)\right\|_{\infty}\left\|p^{e}(t)\right\|-f^{\prime}(t) .
$$

By integrating (83) over $[-r, r]$, we obtain that

$$
\begin{aligned}
& \frac{1}{\mu([-r, r])} \int_{-r}^{r}|<k(t)| h(t)>\mid d \mu(t) \\
& \leq\left\|C^{-1}\left(h^{\prime}+k\right)\right\|_{\infty} \frac{1}{\mu([-r, r])} \int_{-r}^{r}\left\|p^{e}(t)\right\| d \mu(t)-\frac{1}{\mu([-r, r])} \int_{-r}^{r} f^{\prime}(t) d \mu(t) .
\end{aligned}
$$

Since $p^{e}$ is $\mu$-ergodic and $f$ is bounded on $\mathbb{R}$, by taking the limit as $r \rightarrow+\infty$, we deduce that

$$
\left.\lim _{T \rightarrow+\infty} \frac{1}{\mu([-r, r])} \int_{-r}^{r}|<k(t)| h(t)\right\rangle \mid d \mu(t)=0,
$$

then, the function $t \longrightarrow<k(t) \mid h(t)>$ is ergodic. From Lemma 6, we deduce that $t \longrightarrow\|k(t)\|$ is $\mu$-ergodic also.

Consider

$$
g(t)=\left\langle h^{\prime}(t)|h(t)>+<k(t)| h(t)>.\right.
$$

The function $g$ is bounded derivable and

$$
g^{\prime}(t)=\left\langle h^{\prime \prime}(t)\left|h(t)>+\left\|h^{\prime}(t)\right\|^{2}+<k^{\prime}(t)\right| h(t)\right\rangle+\langle k(t)| h^{\prime}(t)>,
$$

by using (81), we have

$$
g^{\prime}(t)=-\langle C h(t)| h(t)>+\left\langle p^{e}(t)\left|h(t)>+\left\|h^{\prime}(t)\right\|^{2}+<k(t)\right| h^{\prime}(t)>.\right.
$$

Since $C$ is negative definite, we obtain that

$$
c\|h(t)\|^{2} \leq\|h\|_{\infty}\left\|p^{e}(t)\right\|+\left\|h^{\prime}\right\|_{\infty}\|k(t)\|+g^{\prime}(t) .
$$

By integrating (85) over $[-T, T]$, we have

$$
\begin{aligned}
& \lim _{r \rightarrow+\infty} \frac{1}{\mu([-r, r])} \int_{[-r, r]} c\|h(t)\|^{2} d \mu(t) \leq\|h\|_{\infty} \lim _{r \rightarrow+\infty} \frac{1}{\mu([-r, r])} \int_{[-r, r]} c\left\|p^{e}(t)\right\| d \mu(t) \\
& +\left\|h^{\prime}\right\|_{\infty} \lim _{r \rightarrow+\infty} \frac{1}{\mu([-r, r])} \int_{[-r, r]}\|k(t)\| d \mu(t)+\lim _{r \rightarrow+\infty} \frac{1}{\mu([-r, r])} \int_{[-r, r]} g^{\prime}(t) d \mu(t) .
\end{aligned}
$$

Since $p^{e}$ and $k$ are $\mu$-ergodic and $g$ is bounded on $\mathbb{R}$, by taking the limit as $T \rightarrow+\infty$, we deduce that

$$
\lim _{r \rightarrow+\infty} \frac{1}{\mu([-r, r])} \int_{[-r, r]} c\|h(t)\|^{2} d \mu(t)=0 .
$$

By using the Cauchy-Schwartz inequality, we deduce that $h=x-y$ is ergodic, then $x$ is pseudo almost periodic and $y$ is the almost periodic component of $x: y=x^{a p}$.

Now, we state a result of existence of pseudo almost periodic solutions.

Denoting $\lambda_{M}$ (resp. $\lambda_{m}$ ) the greatest (resp. smallest) eigenvalues of $B$. Putting $\delta:=\lambda_{M}-\lambda_{m}$ (The spectrum diameter of the operator $B$ ). 
Consider the following hypotheses:

(D1) There exist $x_{0} \in \Omega$ and $R>0$ such that $\bar{B}\left(x_{0}, R\right) \subset \Omega$ and

$$
<p(t)-G\left(x+x_{0}\right) \mid x>\geq \frac{R^{2}}{16} \delta^{2},
$$

for all $t \in \mathbb{R}$ and $x \in \mathbb{R}^{N}$ such that $\|x\|=R$.

(D2) $\Omega=\mathbb{R}^{N},-G$ is $c$-strongly monotone on $\mathbb{R}^{N}$ and $c>\frac{1}{16} \delta^{2}$.

Corollary 33. If we assume that hypothesis (D1) or (D2) is satisfied, then equation (35) has a unique bounded solution on $\mathbb{R}$.

Proof. It follows from Theorem 13 applied with $F(x)=\frac{1}{2}\langle B x \mid x\rangle$ and the proposition 14. Q.E.D.

Theorem 34. Assume $p$ is $\mu$-pseudo almost periodic. If hypothesis (D1) or (D2) is satisfied, then equation (35) has a unique solution $x$ which is bounded on $\mathbb{R}$ and $\mu$-pseudo almost periodic and verifies $\bmod (x) \subset \bmod (p)$. Furthermore, if we denote by $p^{a p}$ (resp. $y$ ) the almost periodic component of $p$ (resp. $x$ ), then $y$ is the almost periodic solution of equation (47).

Remark 35. In the almost periodic case $\left(p \in A P\left(\mathbb{R}, \mathbb{R}^{N}\right)\right)$, the unique bounded solution is almost periodic and verifies the modulus formula.

Proof. From Corollary 33, we obtain the existence and uniqueness of the solution $x$ that is bounded on $\mathbb{R}$ for the equation (35). Firstly we state the existence and uniqueness of the almost periodic $y$ for the equation (47) and $\bmod (y) \subset \bmod \left(p^{a p}\right)$. Since if $p$ verifies (D1) or (D2), then so is for $p^{a p}$. In fact for (D2), it is easily to see that since $D 2$ ) does not depend of the second member $p$. For (D1), it suffices to remark that, if we have

$$
<p(t)-G\left(x+x_{0}\right) \mid x>\geq \frac{R^{2}}{16} \delta^{2},
$$

then, we have

$$
<p^{a p}(t)-G\left(x+x_{0}\right) \mid x>\geq \frac{R^{2}}{16} \delta^{2},
$$

since $t \longrightarrow\left\langle p^{a p}(t)-G\left(x+x_{0}\right)\right| x>$ is the almost periodic component of $t \longrightarrow\left\langle p(t)-G\left(x+x_{0}\right)\right| x>$. From Corollary 33, equation (47) has a unique bounded solution $y$ on $\mathbb{R}$. By using Theorem 16 , the bounded solution $y$ is almost periodic and verifies the formula's modules. Putting $h:=x-y$. to end the proof, it remains to show that $h \in P A P_{0}\left(\mathbb{R}, \mathbb{R}^{N}\right)$. Since $x$ (resp. $y$ ) is a solution of (35) (resp. (47)), then $h$ verifies

$$
h^{\prime \prime}+B h^{\prime}=G(y)-G(x)+p^{e}(t),
$$

where $p^{e}$ denotes the ergodic perturbation of $p$. Then

$$
\begin{aligned}
& <h^{\prime \prime}(t)\left|h(t)>+<B h^{\prime}(t)\right| h(t)> \\
& =<G(y(t))-G(x(t))\left|h(t)>+<p^{e}(t)\right| h(t)>.
\end{aligned}
$$

Putting

$$
\alpha(t):=\left\langle h^{\prime}(t)\left|h(t)>+\frac{1}{2}<B h(t)\right| h(t)\right\rangle,
$$

the function $\alpha$ is derivable and

$$
\alpha^{\prime}(t)=\left\langle G(y(t))-G(x(t))\left|h(t)>+<p^{e}(t)\right| h(t)>+\left\|h^{\prime}(t)\right\|^{2} .\right.
$$

Using the hypothesis (H1) and (86), we obtain that

$$
0 \leq<G(y(t))-G(x(t)) \mid h(t)>\leq\|h\|_{\infty}\left\|p^{e}(t)\right\|+\alpha^{\prime}(t),
$$


where $\|\cdot\|_{\infty}$ denotes the sup norm on $\mathbb{R}$. By integrating (87) over $[-T, T]$, we obtain that

$$
\begin{aligned}
& \frac{1}{2 T} \int_{-T}^{T}|<G(x(t))-G(y(t))| h(t)>\mid d t \\
& \leq\|h\|_{\infty} \frac{1}{2 T} \int_{-T}^{T}\left\|p^{e}(t)\right\| d t+\frac{1}{2 T}[\alpha(t)]_{-T}^{T} .
\end{aligned}
$$

Since $p^{e}$ is ergodic and $\alpha$ is bounded on $\mathbb{R}$, by taking the limit as $T \rightarrow+\infty$, we deduce that

$$
\lim _{T \rightarrow+\infty} \frac{1}{2 T} \int_{-T}^{T}|\langle G(x(t))-G(y(t)) \mid h(t)\rangle| d t=0,
$$

with the function $t \longrightarrow<G(x(t))-G(y(t)) \mid h(t)>$ is ergodic. We deduce from Lemma 8 that $h=x-y$ is ergodic, then $x$ is pseudo almost periodic and $y$ is the almost periodic component of $x: y=x^{a p}$.

Acknowledgement: The authors express their sincere gratitude to the anonymous referee, who carefully read the manuscript and made remarks leading to improvements on the presentation of this paper.

\section{References}

[1] E. Ait Dads, P. Cieutat, and L. Lhachimi, Structure of the set of bounded solutions and existence of pseudo almost periodic solutions of a Liénard equation, Journal of Differential and Integral Equations, Vol. 20, No 7 (2007), 793-813.

[2] E. Ait Dads, O. Arino, and K. Ezzinbi, Pseudo almost periodic solutions of some differential equations in a Banach space, Nonlinear Analysis Theory Methods and Applications,.28, (1997) $\mathrm{N}^{\circ}$ 7, 1141-1155.

[3] E. Ait Dads and K. Ezzinbi, Existence of pseudo almost periodic solution for some abstract semilinear functional differential equation, Dynamic. Systems and Applications. 11 (2002), 493-498.

[4] E. Ait Dads, and O. Arino; Exponential dichotomy and the existence of pseudo almost periodic solutions of some differential equations, Nonlinear Analysis Theory Methods and Applications,27, (1996) № 4, 369-386.

[5] E. Ait Dads, P. Cieutat and S. Fatajou: Pseudo almost automorphic solutions for some nonlinear differential equations: Liénard equations and Hamiltonian systems: International Journal of Evolution equations Volume 3 Issue 4. (2009), 503-524.

[6] M. Brown, Homeomorphisms of two-dimensional manifolds, Houston Journal of Mathematics. 11, (1985), 455-469.

[7] J. Campos and P.J. Torres, On the structure of the set of bounded solutions on a periodic Liénard equation, Proceeding of the American Mathematical Society. 127 (1999), 1453-1462.

[8] P. Cieutat, Maximum principle and existence of almost-periodic solutions of second-order differential systems, Differential and Integral Equations ,17, (2004), 921-942.

[9] P. Cieutat, On the structure of the set of bounded solutions on an almost periodic Liénard equation, Nonlinear Analysis Theory Methods and Applications, 58, (2004), 885-898.

[10] P. Cieutat, Almost periodic solutions of forced vector Liénard equations, Journal of Differential Equations, 209, (2005), 302328.

[11] C. Corduneanu, Almost periodic functions, Wily, New York, 1968. Reprinted, Chelsea, New York, 1989.

[12] T. Diagana, Pseudo almost periodic solutions to some differential equations, Nonlinear Analysis Theory Methods and Applications . 60 (2005), 1277-1286.

[13] Joel Blot, Philippe Cieutat, and Khalil Ezzinbi: Measure theory and pseudo almost automorphic functions: New developments and applications Nonlinear Analysis Theory Methods and Applications, 75 (2012) 2424-2447.

[14] A. M. Fink, Almost periodic differential equations, Lecture Notes in Mathematics, Vol. 377, Springer-Verlag, Berlin-New York, 1974.

[15] P. Habets and L. Sanchez, Periodic solutions of some Liénard equations with singularities, Proceeding of the American Mathematical Society. 109 (1990), 1035-1044.

[16] Hong-xu Li, Huang, Fa-Lun, and Ji-Yong Li, Composition of pseudo almost periodic functions and semilinear differential equations, Journal of Mathematical Analysis and Applications, 255 (2001), 436-446.

[17] A. C. Lazer and S. Solimini, On periodic solutions of nonlinear differential equations with singularities, Proceeding of the American Mathematical Society, 99 (1987), 109-114. 
[18] P. Martínez-Amores and P. J. Torres, Dynamics of a periodic differential equation with a singular nonlinearity of attractive type, Journal of Mathematical Analysis and Applications, 202 (1996), 1027-1039.

[19] R. Yuan, Pseudo-almost periodic solutions of second-order neutral delay differential equations with piecewise constant argument, Nonlinear Analysis Theory Methods and Applications. 41 (2000), 871-890.

[20] C. Zhang ; Pseudo Almost Periodic Functions and Their Applications, Ph.D. thesis, University of Western Ontario, (1992).

[21] C. Zhang, Pseudo Almost-Periodic Solutions of Some Differential Equations, Journal of Mathematical Analysis and Applications,181, (1994) n 1, pp. 62-76.

[22] C. Zhang, A characterization of pseudo almost periodic functions in Fourier analysis, Acta Analysis Functionalis Applicata, 4 (2002), 110-114. 